\title{
Massively Parallel Intelligent Pixel Implementation of a Zerotree Entropy Video Codec for Multimedia Communications
}

\author{
A.M.Rassau*, G.Alagoda, D.Lucas, J.Austin-Crowe, K.Eshraghian \\ Centre for Very High Speed Microelectronic Systems, \\ Edith Cowan University, 100 Joondalup Drive, Joondalup, Perth WA 6027, Australia \\ * Department of Cybernetics, University of Reading, Whiteknights, Reading RG6 6AY, UK \\ *(Currently attached to the Centre for Very High Speed Microelectronic Systems, ECU) \\ Ph.: $+44(0) 1189875123$ \\ Fax.: +44(0)1189318220 \\ E-Mail:amr@cyber.rdg.ac.uk
}

Key words: Parallel architectures, video processing, compression, multimedia applications.

\begin{abstract}
A recently proposed Intelligent Pixel Mobile Multimedia Communicator $\left(\mathrm{IPM}^{3} \mathrm{C}\right)$ integrates the capture, encoding, decoding and display of optical images into a single array of Intelligent Pixel (IP) processors. The real-time video processing required both for compression of the captured images and decompression of the received data stream presents a significant challenge for the required pixel based coding. This paper presents an architecture for the implementation of a massively parallel wavelet based zerotree entropy video codec, designed for implementation on the IP array, capable of fulfilling the very low bit-rate coding requirements for $\mathrm{M}^{3} \mathrm{C}$.
\end{abstract}

The original version of this chapter was revised: The copyright line was incorrect. This has been corrected. The Erratum to this chapter is available at DOI: 10.1007/978-0-387-35498-9_57 


\section{INTRODUCTION}

Integrated image sensor and display devices, capable of real-time image capture and processing, are of significant interest for the future development of interactive multimedia communications devices [1]. A novel single chip image processor capable of real-time simultaneous image capture, processing and display was recently proposed for application to mobile multimedia communications $\left(\mathrm{M}^{3} \mathrm{C}\right)[2]$.

The processor consists of an array of so called Intelligent Pixel (IP) processing elements, shown schematically in Figure 2. Each element of the array incorporates three components. Firstly, a photo-detector (PD) with associated analogue to digital converter (ADC), for the conversion of the incident light level to a suitable input value. Secondly, a localised processor customised for the specific implementation of the parallel video codec algorithm and finally a liquid crystal display for the conversion of the decoded incoming video stream to an optical output [3]. To carry out the required video coding the IP array is used to emulate a mesh network of processing elements such that the inherent parallelism and interconnectivity of the architecture is fully exploited.
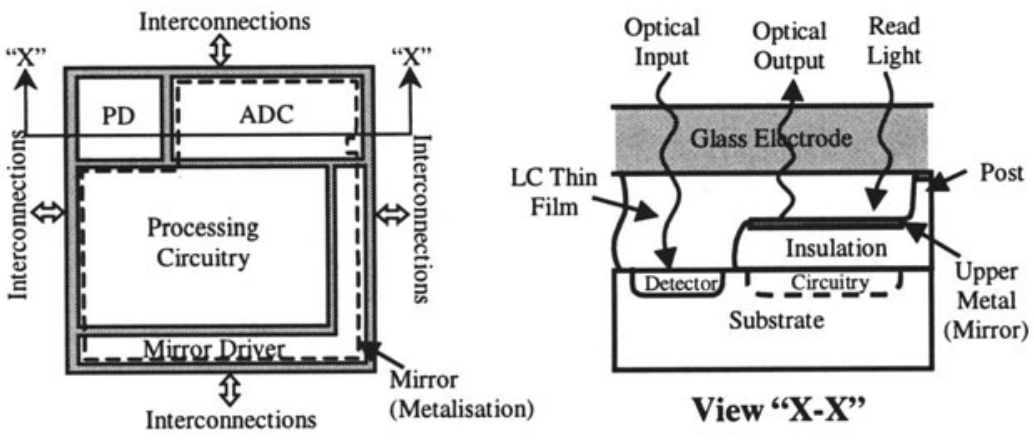

Figure 1. Intelligent Pixel processor

A massively parallel wavelet based video codec algorithm for an IP array processor was recently presented [4]. The proposed IP implementation of the algorithm is capable of real-time very-low-bit-rate video coding with performance comparable to other state-of-the-art codecs and is also highly scalable. These characteristics are crucial to the development of an $\mathrm{M}^{3} \mathrm{C}$ device, and are compatible with the low bit-rate $(\sim 20 \mathrm{~kb} / \mathrm{s})$ recommendation in MPEG-4 and the channel bandwidths of the existing GSM $(23 \mathrm{~kb} / \mathrm{s})$ systems. In this paper a logical architecture for the implementation of this codec within the IP array is described along with the necessary control strategy for embedded parallel array operation. 


\section{VERY LOW BIT-RATE VIDEO COMPRESSION FOR MULTIMEDIA COMMUNICATION}

All of the current video coding standards (ITU, MPEG, etc.) are based on the same general architecture, namely motion-compensated temporal coding coupled with some form of image transform spatial coding. The requirements of the $\mathrm{IPM}^{3} \mathrm{C}$ are for very-low-bit-rate real-time compression of video within tight hardware complexity constraints. Of all the techniques currently in development the most suitable to fulfil this requirement, in terms of performance versus complexity, is almost certainly a combination of the discrete wavelet transform (DWT) coupled with zerotree coding.

Few hardware implementations of zerotree coders have been developed to date, mainly because the structure of the standard embedded zerotree wavelet (EZW) algorithm [5] requires a complex architectural mapping. A new more efficient technique termed zerotree entropy (ZTE) coding has, however, recently been developed by Martucci and Sodagar [6] specifically for very low bit-rate coding of wavelet coefficients. ZTE coding differs from the more traditional zerotree coders in a number of ways that make it suitable for implementation on the parallel IP array. In addition the alphabet of symbols used to classify the tree nodes is changed to one that is capable of performing significantly better for very low bit-rate encoding of video. A very low bit-rate video codec algorithm based on these techniques and suitable for implementation on an IP array is presented in [4]. The system block diagram for the forward path of this algorithm is shown in Figure 3.

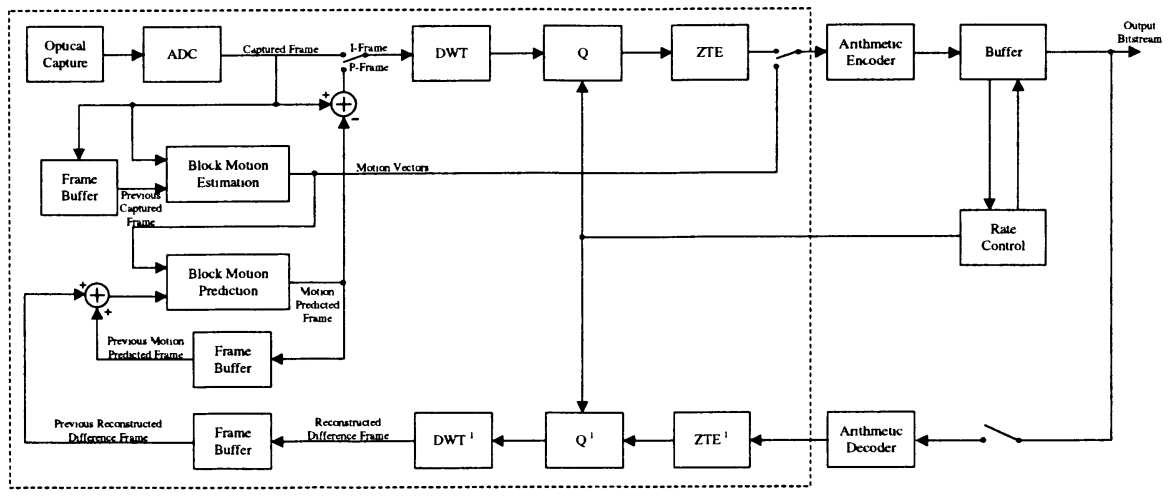

Figure 2. System block diagram of the encoder for the proposed video codec. All functionality prior to the arithmetic coder (shown enclosed within the dashed line) is embedded within the IP array. 


\subsection{Motion Estimation}

The massively parallel IP array is well suited to cope with the large processing demands of a block matching approach to motion estimation. Due to the low resolution of the video in mobile applications, a block size of $8 \times 8$ pixels is sufficient. To achieve very low bit-rate compression the length of the motion vectors must be kept to a minimum to ensure that the motion information does not account for too large a proportion of the available bitbudget. This can be achieved by limiting the search area to a relatively small range. For videoconferencing applications it can be reasonably assumed that the majority of motion to be tracked will be slow moving, thus a small search area is acceptable. A search area of 8 pixels in all four directions from the centre of each block is adequate and by exploiting the parallel processing capabilities of the array a full search within this area can be performed. The sum of absolute differences (SAD) is used as a distortion measure, the block with the lowest SAD being selected for the estimated frame and the appropriate motion vector transmitted. The prediction error calculated from the motion compensated previous reconstructed frame, is further compressed using DWT and ZTE coding to remove spatial correlation.

\section{IPARRAY IMPLEMENTATION}

An array of IPs is uniquely capable of performing highly parallel image processing tasks on images captured in situ within the array. This provides the potential for real-time operation at very high frame rates. The limited hardware complexity that can be realised within each pixel, however, necessitates very efficient architectural mapping of the video coding algorithms.

\subsection{Motion Estimation}

To perform the motion estimation a complete search is carried out in a $16 \times 16$ pixel search area around each motion block in the image to find the closest matching block. The corresponding motion vector for that block is then coded and transmitted. This search can be carried out on all motion blocks in the array in parallel by shifting the entire current frame, through the complete search area, over the previous frame and storing a motion vector in each block corresponding to the point where the distortion measure was lowest. 


\subsection{Discrete Wavelet Transform}

A wavelet transformation is essentially a sub-band decomposition that segments an image into a number of frequency domains at different scales. A complete two-dimensional decomposition can be calculated by sequential convolution of the rows and columns of an image with a pair of suitably chosen quadrature mirror filters (QMFs) followed by half rate sub-sampling at each scale. For a VLSI implementation 1-D FIR filters can be used for these convolutions. Suitably chosen wavelet basis functions are used to derive the filter coefficients. The transform coefficients, $y_{i}$, are thus obtained by convolving the pixel input values, $x_{i}$, with the coefficients, $w_{s}$, of an FIR filter of length $L$ :

$$
y_{i}=\sum_{s=1}^{L} x_{i-s} w_{s}
$$

This convolution can be computed by scaling the image coefficients with each of the filter coefficients in turn and accumulating the sum of these products in each pixel. This can be carried out very efficiently on the IP array as the mesh network of processing elements allows all of the convolutions in one plane to be carried out in parallel.

\subsubsection{Wavelet Transform Using Symmetrical FIR Filters}

An efficient method that can be used to solve the inherent border problem that arises when performing linear convolutions, is to implement a bidirectional data shifting technique as first proposed by Lu [7]. If the FIR filters used to perform the discrete wavelet transform (DWT) are formed from a symmetric kernel of length $2 P+l$ then by using the property $w_{s}=w_{-s}$ the convolution in equation (1) can be rewritten as:

$y_{i}=\sum_{s=1}^{p} x_{i+s} w_{s}+\sum_{s=1}^{p} x_{i-s} w_{s}+x_{0} w_{0}$

Bidirectional shifting of the image data can then be used to calculate the complete convolution in $P$ cycles. Figure 4 illustrates an example of the necessary data movement within one individual row. The pixel input value is replicated and stored in a pair of registers within each IP. In each cycle the data in one of these registers moves to the right while the data in the second moves to the left. After one such bidirectional shift, as shown by the arrows, each IP contains the corresponding $x_{i+s}, x_{i-s}$ and $x_{0}$ ( $x_{0}$ in a third register not shown). This provides symmetry between the first and last rows of the array 
and thus avoids the reconstruction error on the image border by removing the discontinuity at the edges.

In addition to solving the finite border problem this scheme lends itself particularly well to an efficient hardware implementation. Due to the symmetric nature of the filters used, only half of the filter coefficients are required to carry out the convolution. In fact the convolution sum can very easily be calculated from the bidirectionally shifted data. $x_{i-s}$ is the right shifted data, shown at the top of each pixel in Figure 4, and $x_{i+s}$ is the left shifted data, shown at the bottom. After each shift these two filtered data values need only be multiplied with the next positive filter coefficient and the sum of both results accumulated in each of the processors [8].

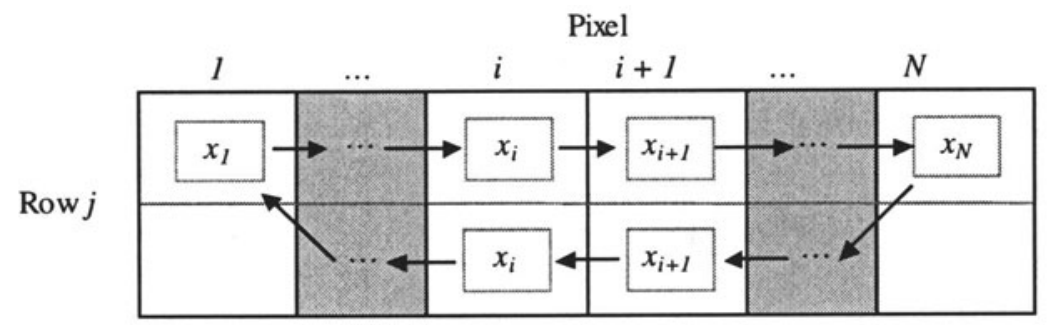

Figure 3. Schematic diagram showing data movement for the bidirectional shifting method

\subsubsection{Binary Wavelet Filters and ZTE Coding}

When considering low complexity hardware implementations the precision with which the filter coefficients can be represented must be considered. Integer approximations need to be made which will lead to systematic errors during reconstruction. These errors can, however, be avoided if filters with binary representable coefficients $[9,10]$ are used. The coefficients can then be represented by powers of 2 and the scaling required for the convolution calculations can be carried out using single-bit shifts.

Due to the limited precision available for the calculations within the IP architecture binary wavelet filters have been shown to perform as well if not better for hardware implementations of wavelet based ZTE coders than the standard real-valued filters such as Daubechies' [11]. Because the simple binary filters have inferior spatial-frequency localisation properties, they tend to produce relatively large coefficients in the high frequency sub-bands. This does not adversely affect ZTE coding to any great extent, however, due to the fact that all significant coefficients are transmitted in one pass unlike the multiple passes used with the more standard EZW coding

The use of binary representable filters allows the convolution operations necessary for the computation of the DWT to be carried out using integer 
arithmetic. A complete multi-scale DWT can thus be computed on the IP array using a single bit-serial adder accumulator along with two additional registers to store the current frame for left and right shifting.

\subsubsection{Nucleic Coefficient Storage}

After all of the convolutions for a particular scale of the decomposition have been carried out, four frequency sub-bands are obtained (LL, LH, HL and $\mathrm{HH}$ ). For the next scale it is only the low-low (LL) filtered transform coefficients that are further decomposed. A nucleic scheme can be used to efficiently store these sub-band coefficients within the IP array. As the decomposition progresses to each subsequent scale only certain nuclei, which contain the data required at that scale remain active [2]. The rest of the pixels retain the data from the previous scales but are put into a bypass mode making them transparent to the decomposition at the current scale. The resulting arrangement is illustrated in Figure $5 . \mathbf{L L}_{\mathbf{s}}{ }^{\mathbf{x y}}$ represents a low-low filtered coefficient at scale $\mathbf{s}$ of the decomposition from cell $\mathbf{x}, \mathbf{y}$.

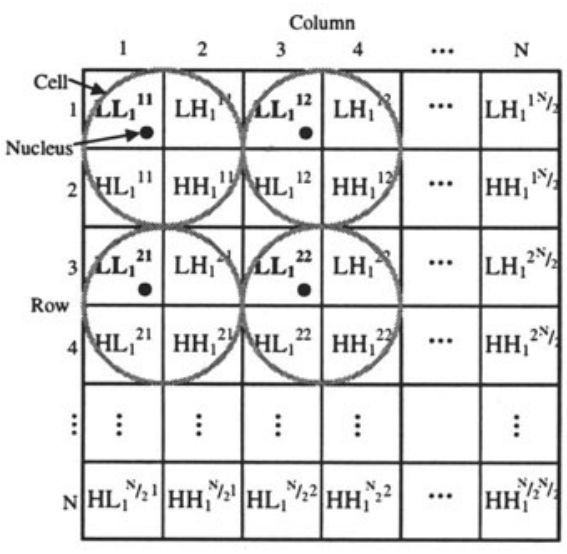

First scale of decomposition

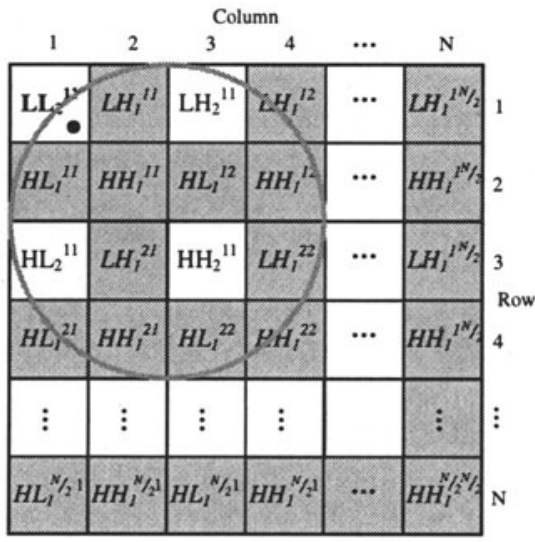

Second scale of decomposition

Figure 4. Two scale decomposition of an $\mathrm{NxN}$ image in the nucleic scheme. $\mathrm{LL}_{\mathrm{s}}{ }^{\mathrm{xy}}$ represents a low-low filtered coefficient obtained at scale $s$ of the decomposition in cell $x, y$

This allows the decomposition at any scale to be carried out with the same time complexity and has the additional advantage that at the end of the transform the coefficients are ordered together into wavelet blocks facilitating the implementation of the ZTE coder. 


\subsection{Zerotree Entropy Coder}

ZTE coding is based on, but differs significantly from, the well-known EZW algorithm. Like EZW, the ZTE algorithm exploits the self-similarity inherent in the wavelet transform of image frames to predict the location of information across wavelet scales. The wavelet coefficients are quantised and zerotrees are used to reduce the number of bits required to represent the wavelet trees. In ZTE coding, the coefficients of each wavelet tree rooted in the lowest band are rearranged to form a wavelet block. This wavelet block provides a direct association between the wavelet coefficients and what they represent spatially in the frame. Related coefficients at all scales and orientations are included in each block. As previously discussed the transform coefficients are already arranged into these wavelet blocks after the nucleic wavelet transform so no rearrangement is necessary prior to ZTE coding.

To perform ZTE coding each wavelet block is first adaptively quantised according to scene content and frequency band as well as the desired bit-rate. The extreme quantisation required to achieve a very low bit-rate results in a high proportion of zero coefficients. The wavelet trees can thus be efficiently represented and coded by scanning each tree depth-first from the root in the low-low sub-band through the children and assigning either a zerotree root $(Z T R)$, valued zerotree root (VZ) or value $(V)$ symbol to each appropriately. A zerotree root exists at any node where the coefficient is zero and all the node's children are zerotrees. Zerotrees do not need to be scanned further because it is known that all coefficients in these trees have amplitude zero. A valued zerotree root is a node where the coefficient has a non-zero amplitude but all four children are zerotree roots, again the scan of the tree can stop at this symbol. A value symbol identifies a coefficient that has some non-zero descendants; its amplitude can be zero or non-zero.

\section{ARCHITECTURAL MAPPING}

The approaches outlined above allow a hardware implementation of the proposed video codec to be achieved very efficiently. Each pixel need only contain a single bit-serial adder-accumulator, a number of shift registers for frame buffering and coefficient storage, a multiplexer to route signals between pixels and a small amount of additional control logic. 


\subsection{Discrete Wavelet Transform}

Figure 7 outlines the logical architecture necessary to carry out the DWT using a symmetric binary representable wavelet filter. Due to the limited space available within the IPs all arithmetic operations are performed in a bit-serial fashion. The registers in each pixel need to operate as bi-directional shifters in order to perform the scaling operations. Shift-right is the inherent operation and will occur at every clock cycle, shift-left is equivalent to a noshift-right operation for one clock cycle. The 4-way multiplexer in each of the pixels is used to select as input to the pixel the output from any one of the neighbouring pixels. In order to carry out the convolutions required for the DWT Reg1 and Reg2 alternately load data from the left or right neighbouring pixels respectively or pass data to the adder-accumulator. This allows the summation and the data shifting operations to be carried out in parallel.

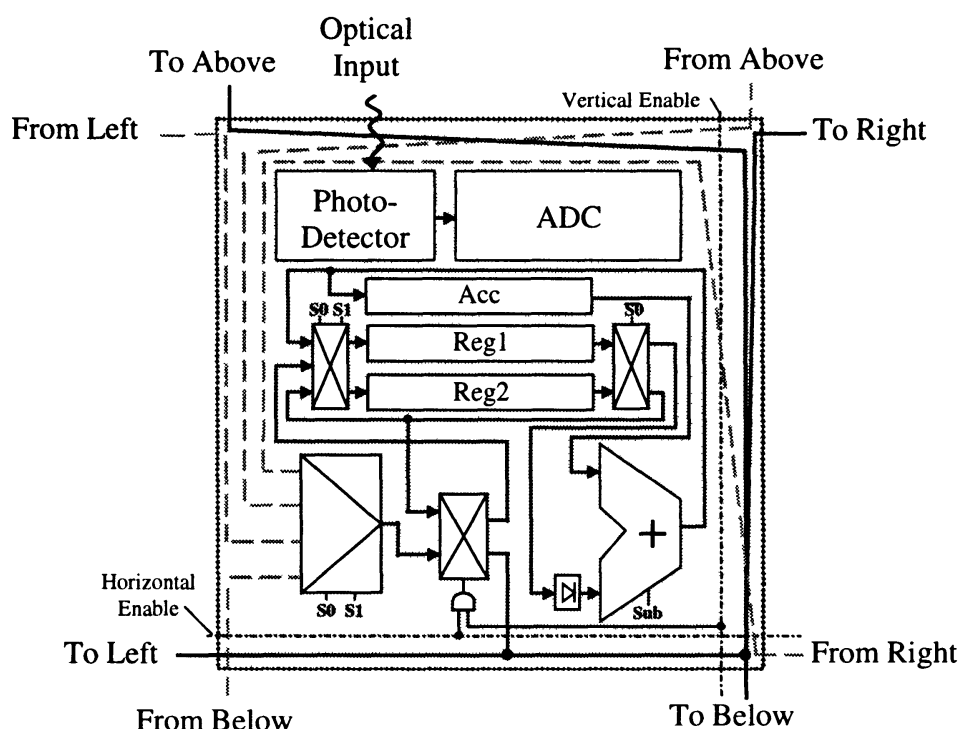

Figure 5. Logical architecture within each pixel for computation of the DWT

The decimation required after each scale of the transform is inherent in the ordering of the coefficients in the pixels. All odd numbered rows or columns of pixels perform the low pass filtering and all even numbered row or columns the high pass filtering. At the end of each scale all of the coefficients are thus arranged in the nucleic scheme introduced above. To perform the transform at the next scale the even rows and columns containing all the high frequency sub-bands need only be disabled such that 
the convolution operations can be performed as before on the low-low subband. In order to disable the high pass pixels they are placed into a bypass or transparent mode so that data can be passed directly through them. In active pixels the 4-way multiplexer is connected to one of Regl or Reg2 and the output from that register is connected to the output bus. When a pixel is switched into the bypass or transparent mode of operation the 4-way multiplexer is connected directly back to the output bus in the same pixel.

Each pixel in the array is controlled by a select line that determines whether it functions in a low pass or a high pass filter mode. These select lines run all the way through every row and column. At the start of the forward transform all even numbered rows and columns are set to operate in the high pass mode and the odd numbered rows and columns in the low pass mode. At the end of the scale all pixels that were in the high pass mode are switched into bypass mode leaving only the pixels containing the low-low filtered coefficients active. For the next scale and all subsequent scales the first row and column always remain in the low pass mode. Subsequently every alternate row or column is switched into high pass mode, noting that the bypass mode pixels are effectively transparent. The algorithm is completely symmetric so for the inverse transform this process is simply carried out in reverse.

\subsection{Zerotree Entropy Coder}

The arrangement produced by the nucleic DWT enables a ZTE processor to be designed that can independently process each wavelet block in parallel. To carry out ZTE coding each wavelet block is first adaptively quantised and the significance, $S$, of each pixel determined. A pixel is significant if the magnitude of its quantised coefficient is greater than zero. Child significance, $C$, is then derived from this. This signal propagates through the entire wavelet block, it is high if any descendants of a pixel are significant or have significant descendants. The pixels at the lowest level have no children hence $C$ is always zero for these. Parent significance, $P$, is further derived from this, it is high if a pixels parent or any of its siblings are significant.

These three signals are used to derive two status bits, $A$ and $B$, which correspond to one of four states indicating whether the pixel is a zerotree root (ZTR), a valued zerotree root (VZ), a value (V) or does not need to be transmitted as it is part of zerotree (DNT). Figure 8 shows the logical architecture required in each pixel to derive the necessary significance information. 


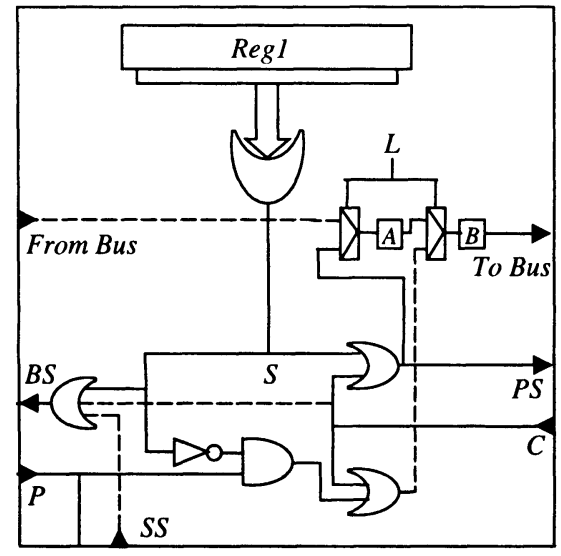

(a)

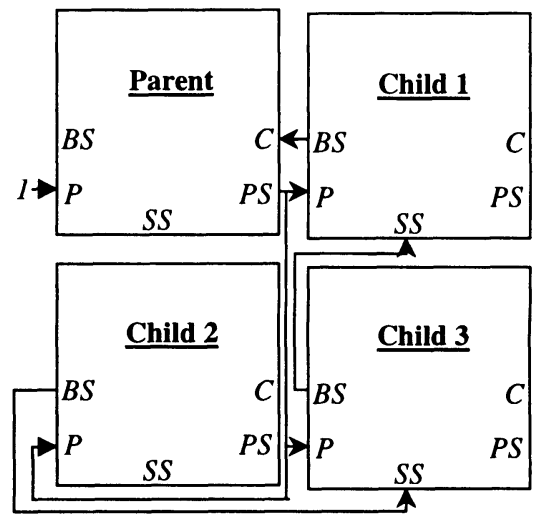

(b)

Figure 6. (a) Logical architecture for the hardware implementation of the ZTE coder and

(b) Parent-child interconnections for the first wavelet scale.

The SS signal routes the significance information between sibling pixels within one level of a wavelet block. The BS signal is high if either the pixel, any of its siblings or their children are significant. The PS signal is high if the pixel or any of its children are significant.

\subsection{Control Strategy}

The limited space available for processing within each individual pixel in the array necessitates some degree of external control. Each IP contains one additional register to store a series of control bits that define the state of the multiplexers and other logic within the IP to appropriately route data between and within the pixels. Appropriate control words generated by a state machine external to the array are thus loaded into the pixels to carry out all of the necessary stages of the video coding algorithm, i.e. motion compensation, forward WT and ZTE coding for captured frames, followed by ZTE decoding, inverse WT and motion prediction for simultaneous display.

\section{CONCLUSION}

The logical architecture and control strategy required for the implementation of a very low bit-rate video codec on an IP array has been described. Due to the massively parallel nature of the IP array the architecture is capable of operating in real-time at very high frame-rates. A complete three-scale forward wavelet transformation followed by ZTE coding can be performed in less than 300 clock cycles irrespective of image size. VHDL hardware simulations have been performed, which verify that 
the hardware implementation of the video codec performs similarly to the ZTE codec presented in the literature. Results have shown that this low complexity codec is capable of performing comparably to other state-of-theart very low bit-rate coders for scenes with relatively low levels of motion, as would be common for a personal communication application.

\section{ACKNOWLEDGEMENTS}

Contributions provided by the Australian Research Council and the support of the Centre for Very High Speed Microelectronic Systems, Edith Cowan University are gratefully acknowledged.

\section{REFERENCES}

[1] V.O.K.Li and X.Qiu, "Personal communication systems (PCS)", Proc. of IEEE, Vol. 83, No. 9, pp. 1210-1243, September 1995.

[2] A.M.Rassau, T.C.B.Yu, H.N.Cheung, S.W.Lachowicz, K.Eshraghian, W.A.Crossland and T.D.Wilkinson, "Smart pixel implementation of a 2D parallel nucleic wavelet transform for mobile multimedia communication", Design and Test Conference, Paris, Feb. 1998.

[3] N.Collings, W.A.Crossland, P.J.Ayliffe, D.G.Vass and I.Underwood, "Evolutionary development of advanced liquid crystal spatial light modulators", Applied Optics, Vol. 28, No. 22, November 1989.

[4] A.M.Rassau, G.Alagoda and K.Eshraghian, "Massively parallel wavelet based video codec for an intelligent pixel mobile multimedia communicator", (submitted to the $5^{\text {th }}$ Int. Sym. On Signal Processing and its Applications, Brisbane, Australia, August 1999).

[5] J.M.Shapiro, "Embedded image coding using zerotrees of wavelet coefficients", IEEE Trans. On Signal Processing, Vol. 41, No. 12, pp. 3445-3462, December 1993.

[6] S.A.Martucci and I.Sodagar, "Zerotree entropy encoding of wavelet coefficients for very low bit rate video", Proc. of the IEEE Int. Conf. Image Processing, Switzerland, September 1996.

[7] J.Lu, "Computation of 2-D wavelet transform on the massively parallel computer for image processing", Proc. of the $7^{\text {th }}$ IEEE Multi-D Signal Processing Workshop, Lake Placid, NY, September 1991.

[8] A.M.Rassau, K.Eshraghian, H.Cheung, S.W.Lachowicz, T.C.B.Yu, W.A.Crossland and T.D.Wilkinson, "Wavelet transform algorithms for smart pixel Opto-VLSI implementation", Int. Conf. on Computational Engineering and Systems Applications, Tunisia, April 1998.

[9] E.L.Schwartz, A.Zandi and M.Boliek, "Implementation of compression with reversible embedded wavelets", Proc. of the SPIE 40 ${ }^{\text {th }}$ Annual Meeting, Vol. 2564-04, July 1995.

[10] G.Strang and T.Nguyen, "Wavelets and filter banks", Wellesley Cambridge Press, 1996.

[11] A.M.Rassau, Soo-Ik Chae and K.Eshraghian, "Simple binary wavelet filters for efficient VLSI implementation”, Elec. Letters, Vol. 35, No. 7, pp. 555-557, April 1999. 